Massimo Girardis
Andrea Cossarizza

\title{
A Janus role for MerTK in the outcome of septic shock
}

Received: 26 July 2013

Accepted: 6 September 2013

Published online: 3 October 2013

(C) Springer-Verlag Berlin Heidelberg and ESICM 2013

\section{Girardis ( $)$ A. Cossarizza}

Intensive Care and Immunology, Department of Surgery, Medicine, Dentistry and Morphological Sciences, University of Modena and Reggio Emilia, Via del Pozzo 71, 41125 Modena, Italy

e-mail: girardis.massimo@unimore.it

Severe sepsis remains a clinical challenge today, even in developed countries, because of the increased age of the patients and the high burden of antibiotic resistance [1]. In addition, in recent years, we have become increasingly aware that, even in the early stages of sepsis, a high proportion of patients experience a severe dysfunction of native and adaptive immunity, which is closely related to patients' outcome [2]. In this context, understanding the functionality of receptors able to modulate the immune response, and in particular to downregulate inflammation, has great importance for designing new therapeutic strategies. Among these molecules, a main role is occupied by the TAM family of receptor tyrosine kinases, which is formed by three members, i.e., Tyro3, AxI, and Mer, that have two vitamin $\mathrm{K}$-dependent ligands, i.e., the growth arrest-specific protein (Gas)6 and Protein S [3]. In the September 2013 issue of Intensive Care Medicine, Guignant et al. [4] explored by an elegant experimental study the expression of TAM receptors in circulating immune cells in patients with septic shock and after trauma, and in healthy volunteers. A significant early alteration of MerTK expression on monocytes and neutrophils was observed in patients with septic shock, and a persistent MerTK overexpression after septic shock was associated with adverse outcome. The ex vivo analysis showed also a different
MerTK surface expression of healthy monocytes after $48 \mathrm{~h}$ incubation with plasma from septic or trauma patients, supporting the difference in TAM expression between infection-induced inflammation and sterile inflammation (i.e., trauma). The study provides new important data on the mechanisms of immune response in sepsis, but the true reasons for the observed alterations in MerKT expression and their role in the complex pathophysiological context of sepsis remain to be clarified.

TAM receptors are under the control of Toll-like receptors (TLR), and are widely expressed by cells of the innate immune system [5]. Activation of TAM receptors by their ligands triggers a cascade of intracellular events that culminate in the inhibition of nuclear factor-kappaB (NF$\kappa \mathrm{B})$, a crucial proinflammatory molecule, and results in downregulation of inflammation [6]. When expressed by monocytes and granulocytes, these receptors (and in particular MerTK) can also mediate the phagocytosis of apoptotic cells [7], which is fundamental in order to remove dead cells without triggering inflammation. Several data now exist that underline the importance of MerTK activation in the elimination of potential autoantigens derived by apoptotic cells, in the inhibition of the production of proinflammatory cytokines, and in the prevention of the expansion of autoreactive lymphocytes [8-10]. The functional impairment of MerTK promotes not only autoimmunity but also atherosclerosis, while its overexpression is linked to poor prognosis in cancer [11].

The strong relationships existing between TAM receptors and a proinflammatory molecule such as tumor necrosis factor-alpha (TNF- $\alpha)$ are reinforced by the observation that they can interact with a common protein, the matrix metalloproteinase TNF- $\alpha$ converting enzyme, also known as "a disintegrin and a metalloproteinase 17 " (ADAM17). In order to provide the mature, soluble, secretable form of TNF- $\alpha$, ADAM17 cleaves the membrane form of this important proinflammatory cytokine [12]. Interestingly, ADAM17 is also able to cleave the 
membrane forms of AxI and Mer [13]. Such cleavage results not only in the internalization of the receptors and in the transport off the membrane for recycling, but also in the release of the shortened soluble forms of AxI and Mer that can act as decoy receptor and bind Gas6 and Protein S. In turn, the capture of these ligands decreases the amount of molecules that should bind the membrane forms of TAM receptors, a crucial event to downregulate inflammation. Indeed, in chronic active lesions from patients with multiple sclerosis, it has been shown that the increase in the soluble forms of these receptors due to augmented ADAM17 activity is associated with a sequestration of Gas6 and subsequent increased inflammation [14]. In a mouse model of inflammation, exposure of lungs to lipopolysaccharide (LPS) enhanced the production of soluble Mer [15], whereas the upregulation of membrane Mer was able to prevent the inflammatory cascade during lung injury [16].

Considering the aforementioned observations and the anti-inflammatory role of TAM receptors, the persistent presence of high amounts of membrane Mer in monocytes and granulocytes from septic shock patients with adverse outcome could seem a paradox. However, this phenomenon could be explained by taking into account the possible activity of ADAM17, and its capacity to produce soluble forms of TAM receptors (Fig. 1). Indeed, considering that ADAM17 is activated by LPS [17], it might be that the ratio between soluble and membrane forms of TAM receptors (or even the absolute amount of soluble receptor) becomes crucial for regulating the antiinflammatory action of Gas6 and Protein S. In this perspective, the increase in the membrane form of Mer described by Guignant et al. could be considered a compensatory, antiinflammatory mechanism triggered by innate cells to reduce damage. However, prolonged ADAM17 activity could cause the accumulation of the soluble form of Mer, which captures the aforementioned antiinflammatory molecules and inhibits their action. Thus, one can hypothesize that those patients who have high basal ADAM17 activity are at high risk for a negative outcome. Further studies, including the quantification of soluble MerTK, are urgently required to verify whether this hypothesis is correct, to confirm the importance of persistent MerTK expression as a novel biomarker, and, eventually, to explore new therapeutic strategies for septic shock based upon ADAM17 inhibition.

In fact, several inhibitors of metalloprotease have already been synthesized and tested in different

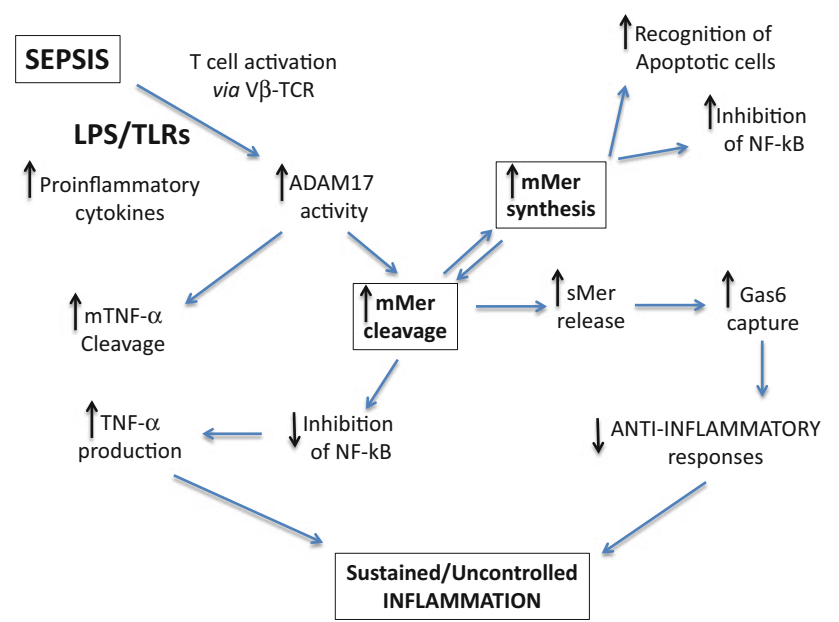

Fig. 1 Possible mechanism linking the persistent increase of MerTK to the poor outcome of septic shock. Sepsis, through the induction of a potent inflammatory response (mediated by LPS/ Toll-like receptors, cytokine, and unspecific, massive $\mathrm{T}$ cell activation), causes an increased activity of the matrix metalloproteinase TNF- $\alpha$ converting enzyme, known as ADAM17. This enzyme not only cleaves the membrane form of TNF- $\alpha$, but also cleaves the membrane form of the TAM receptor Mer. Soluble Mer can act as a decoy receptor and capture the growth arrest-specific protein (Gas)6, whose binding to the membrane form of Mer would trigger an antiinflammatory response. The increased amount of membrane Mer on innate immune cells usually favors the recognition and clearance of apoptotic cells and the inhibition of $\mathrm{NF}-\kappa \mathrm{B}$ (thus exerting an antiinflammatory effect). However, such increase could provide more substrates for the action of ADAM17, which, in a non-mutually exclusive manner, could result in an increase of soluble Mer. Thus, it can be hypothesized that the relative balance between the two forms of Mer, as well as the amount of soluble Mer, could influence the outcome of septic shock

pathological settings. Unfortunately, the first generation and second generations of inhibitors (such as batimastat, ilomastat, marimastat, and prinomastat) showed problems related to their bioavailability, pharmacokinetics, and low selectivity, and were stopped in phase I or II for toxicity problems [18]. However, due to the large theoretical therapeutic potential, it is likely that a new generation of compounds will prove their efficacy very soon and, thus, may become a new useful therapeutic option in patients with severe sepsis and specific alterations in TAM or metalloprotease expression.

Conflicts of interest The authors have no competing interests to declare.

\section{References}

1. Angus DC, van der Poll T (2013) Severe sepsis and septic shock. N Engl J Med 369:840-851
2. Hotchkiss RS, Monneret G, Payen D (2013) Immunosuppression in sepsis: a novel understanding of the disorder and a new therapeutic approach. Lancet Infect Dis 13:260-268
3. Linger RM, Keating AK, Earp HS, Graham DK (2008) TAM receptor tyrosine kinases: biologic functions, signaling, and potential therapeutic targeting in human cancer. Adv Cancer Res 100:35-83 
4. Guignant C, Venet F, Planel S, Demaret J, Gouel-Chéron A, Nougier C, Friggeri A, Allaouchiche B, Lepape A, Monneret G (2013) Increased MerTK expression in circulating innate immune cells of patients with septic shock. Intensive Care Med 39:1556-1564. doi:10.1007/s00134-013-3006-9

5. Rothlin CV, Ghosh S, Zuniga EI, Oldstone MB, Lemke G (2007) TAM receptors are pleiotropic inhibitors of the innate immune response. Cell 131:1124-1136

6. Alciato F, Sainaghi PP, Sola D, Castello L, Avanzi GC (2010) TNF-alpha, IL-6, and IL-1 expression is inhibited by GAS6 in monocytes/macrophages. J Leukoc Biol 87:869-875

7. Lemke G, Rothlin CV (2008) Immunobiology of the TAM receptors. Nat Rev Immunol 8:327-336

8. Shao WH, Zhen Y, Eisenberg RA, Cohen PL (2009) The Mer receptor tyrosine kinase is expressed on discrete macrophage subpopulations and mainly uses Gas6 as its ligand for uptake of apoptotic cells. Clin Immunol 133:138-144

9. Zizzo G, Hilliard BA, Monestier M, Cohen PL (2012) Efficient clearance of early apoptotic cells by human macrophages requires M2c polarization and MerTK induction. J Immunol 189:3508-3520
10. Khan TN, Wong EB, Soni C, Rahman ZS (2013) Prolonged apoptotic cell accumulation in germinal centers of Mer-deficient mice causes elevated B cell and CD4+ Th cell responses leading to autoantibody production. J Immunol 190:1433-1446

11. Verma A, Warner SL, Vankayalapati H, Bearss DJ, Sharma S (2011) Targeting Axl and Mer kinases in cancer. Mol Cancer Ther 10:1763-1773

12. Moss ML, Jin SL, Becherer JD, Bickett DM, Burkhart W, Chen WJ, Hassler D, Leesnitzer MT, McGeehan G, Milla M, Moyer M, Rocque W, Seaton T, Schoenen F, Warner J, Willard D (1997) Structural features and biochemical properties of TNF-alpha converting enzyme (TACE). J Neuroimmunol 72:127-129

13. Thorp E, Vaisar T, Subramanian M, Mautner L, Blobel C, Tabas I (2011) Shedding of the Mer tyrosine kinase receptor is mediated by ADAM17 protein through a pathway involving reactive oxygen species, protein kinase $\mathrm{C} \delta$, and $\mathrm{p} 38$ mitogen-activated protein kinase (MAPK). J Biol Chem 286:33335-33344

14. Weinger JG, Omari KM, Marsden K, Raine CS, Shafit-Zagardo B (2009) Upregulation of soluble Axl and Mer receptor tyrosine kinases negatively correlates with Gas6 in established multiple sclerosis lesions. Am J Pathol 175:283-293
15. Lee YJ, Han JY, Byun J, Park HJ, Park EM, Chong YH, Cho MS, Kang JL (2012) Inhibiting Mer receptor tyrosine kinase suppresses STAT1, SOCS1/3, and NF- $\kappa \mathrm{B}$ activation and enhances inflammatory responses in lipopolysaccharide-induced acute lung injury. J Leukoc Biol 91:921-932

16. Choi JY, Park HJ, Lee YJ, Byun J, Youn YS, Choi JH, Woo SY, Kang JL (2013) Upregulation of Mer receptor tyrosine kinase signaling attenuated lipopolysaccharide-induced lung inflammation. J Pharmacol Exp Ther 344:447-458

17. Arndt PG, Strahan B, Wang Y, Long C, Horiuchi K, Walcheck B (2011) Leukocyte ADAM17 regulates acute pulmonary inflammation. PLoS ONE 6:e19938

18. Murumkar PR, DasGupta S, Chandani SR, Giridhar R, Yadav MR (2010) Novel TACE inhibitors in drug discovery: a review of patented compounds. Expert Opin Ther Pat $20: 31-57$ 